\title{
Presentations of surface braid groups by graphs
}

\author{
by \\ Paolo Bellingeri (Pisa) and \\ Vladimir Vershinin (Montpellier and Novosibirsk)
}

\begin{abstract}
We extend and generalise Sergiescu's results on planar graphs and presentations for the braid group $B_{n}$ to other topological generalisations of $B_{n}$.
\end{abstract}

1. Introduction. Some years after the definition of braid groups by Artin, Zariski $[35,36]$ introduced new groups, which were a natural extension of both the classical braid group $B_{n}$ and the fundamental group of a surface. These groups were then "rediscovered" in the 60's in the study of configuration spaces [17] and called surface braid groups.

A possible definition of such groups is the following. Let $\Sigma$ denote an orientable, connected, compact surface possibly with boundary components. Fix a set $\mathcal{P}=\left\{P_{1}, \ldots, P_{n}\right\}$ of $n$ distinct points in the interior of $\Sigma$. A geometric braid on $\Sigma$ based at $\mathcal{P}$ is a collection $\beta=\left(\psi_{1}, \ldots, \psi_{n}\right)$ of $n$ disjoint paths on $\Sigma \times[0,1]$, called strands of $\beta$, such that the $i$ th strand runs monotonically in $t$ in $[0,1]$ from the point $\left(P_{i}, 0\right)$ to some point $\left(P_{j}, 1\right)$. Two braids are considered equivalent if they are isotopic. The usual product of paths defines a group structure on the equivalence classes of braids. This group, denoted by $B_{n}(\Sigma)$, does not depend on the choice of $\mathcal{P}$ and is called the braid group on $n$ strands on $\Sigma$. The braid group of the disk $B_{n}\left(D^{2}\right)$ is isomorphic to the classical braid group $B_{n}$ and it embeds in a canonical way in $B_{n}(\Sigma)$, when $\Sigma$ is a surface of genus $g \geq 1$. Therefore we can consider the standard generators of the braid group $\sigma_{1}, \ldots, \sigma_{n-1}$ as elements of $B_{n}(\Sigma)$. On the other hand, the group $B_{1}(\Sigma)$ is isomorphic to the fundamental group of $\Sigma$.

Surface braid groups are related to mapping class groups and links in 3 -manifolds [7]. In the last years the interest in these groups has grown

2000 Mathematics Subject Classification: Primary 20F36; Secondary 20 F05.

Key words and phrases: braids, surface braids, graphs.

The second author was supported by CNRS-NSF grant No 17149 and INTAS grant No 03-5-3251. 
considerably (see for instance $[19,20,23,25,27,28]$ ) and several properties of braid groups (and their singular extensions) have been generalised to surface braid groups. In particular, some results on braids and links have been extended to surface braids and links in 3-manifolds [22, 26, 31], and new features have been discovered $[4,16]$.

Sergiescu $[29,30]$ showed how to associate to any planar, connected graph with $n$ vertices, without loops or intersections, a presentation for the braid group $B_{n}$. To each edge $e$ of the graph he associated the braid $\beta_{e}$ which is a clockwise half-twist along $e$ (see Figure 1). Sergiescu provided a complete set of relations using this set of generators for $B_{n}$. Afterwards, Birman, Ko and Lee [9] extended this result to inner-complete graphs in order to give a new proof for the conjugation problem in $B_{n}$. Recently Han and Ko [24] showed that it is possible to associate braid group presentations to a more general family of graphs (linearly spanned graphs) containing the above graphs. We also recall that these presentations turned out to be useful in other related contexts (see for instance [5] and [6]).
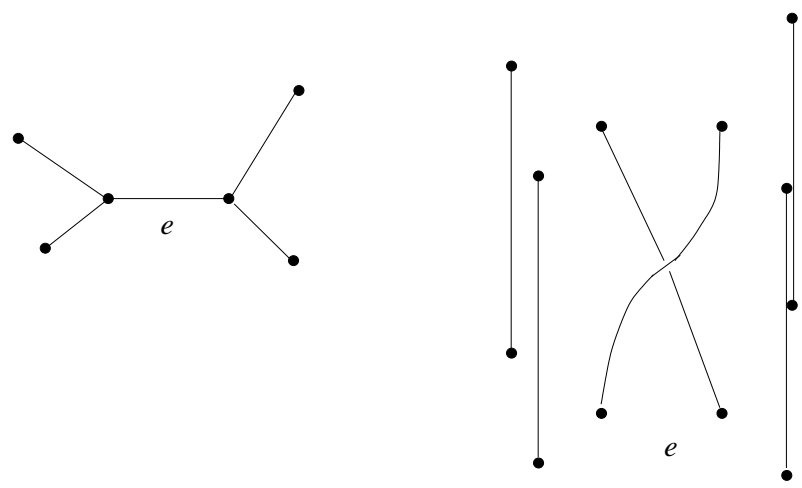

Fig. 1. Edges and geometric braids

The aim of this paper is to provide some analogous results for surface braid groups. Let $\Gamma$ be a graph on an orientable surface $\Sigma$. The graph $\Gamma$ is called normal if it is connected, finite and has no loops or intersections. Let $\Gamma$ be a normal graph on $\Sigma$. Let $S(\Gamma)$ be the set of vertices of $\Gamma$. We associate to the edges of $\Gamma$ the corresponding geometric braids on $\Sigma$ (Figure 1) and we define $B_{\Gamma}(\Sigma)$ as the subgroup of $B_{|S(\Gamma)|}(\Sigma)$ generated by these braids. In Sections 2 and 3 we consider graphs on the sphere and we prove that if $\Gamma$ is a normal graph on the sphere then the set $X_{\Gamma}=\{\sigma \mid \sigma$ is an edge of $\Gamma\}$ is a set of generators for $B_{|S(\Gamma)|}\left(S^{2}\right)$ and thus the group $B_{\Gamma}\left(S^{2}\right)$ coincides with $B_{|S(\Gamma)|}\left(S^{2}\right)$. Moreover, we provide a complete system of relations (Theorem 2.1). In particular, we have the following proposition. 
Proposition 1.1. Let $\Sigma$ be an oriented surface such that $\pi_{1}(\Sigma) \neq 1$ and let $\Gamma$ be a normal graph on $\Sigma$. Then $B_{\Gamma}(\Sigma)$ is a proper subgroup of $B_{|S(\Gamma)|}(\Sigma)$.

In Section 4 we introduce planar graphs with coloured edges and we show how to find presentations via graphs for the braid group on the annulus $B_{n}$ (Ann) (Theorem 4.1). In Section 5 we provide similar results for singular braids on the disk and on the annulus.

\section{Sphere braid group presentations via graphs}

2.1. Definitions and statement of the main theorem. Unless otherwise stated, in Sections 2 and $3, \Gamma$ denotes a normal graph on $S^{2}$. We need some preliminary definitions. Suppose that $\Gamma$ is not a tree. The set $S^{2} \backslash \Gamma$ is the disjoint union of a finite number of open disks $D_{1}, \ldots, D_{m}, m>1$. The boundary of $D_{j}$ on $S^{2}$ is a subgraph $\Gamma\left(D_{j}\right)$ of $\Gamma$. We choose a point $O$ in the interior of $D_{j}$, and an edge $\sigma$ of $\Gamma\left(D_{j}\right)$ with vertices $v_{1}, v_{2}$. We suppose that the triangle $O v_{1} v_{2}$ is oriented anticlockwise. We denote $\sigma$ by $\sigma\left(e_{1}\right)$. We define the pseudocycle associated to $D_{j}$ to be the sequence of edges $\sigma\left(e_{1}\right) \cdots \sigma\left(e_{p}\right)$ such that:

- if the vertex $v_{j+1}$ is not univalent, then $\sigma\left(e_{j+1}\right)$ is the first edge to the left of $\sigma\left(e_{j}\right)$ (we consider $\sigma\left(e_{j}\right)$ going from $v_{j}$ to $v_{j+1}$ ) and the vertex $v_{j+2}$ is the other vertex adjacent to $\sigma\left(e_{j+1}\right)$;

- if the vertex $v_{j+1}$ is univalent, then $\sigma\left(e_{j+1}\right)=\sigma\left(e_{j}\right)$ and $v_{j+2}=v_{j}$;

- $v_{p+1}=v_{1}$.

Let $\gamma=\sigma\left(e_{1}\right) \cdots \sigma\left(e_{p}\right)$ be a pseudocycle of $\Gamma$. Let $i=1, \ldots, p$. If $\sigma\left(e_{i}\right)=$ $\sigma\left(e_{j}\right)$ for some $j \neq i$, then we say that

- $\sigma\left(e_{i}\right)$ is the start edge of a reverse if $j=i+1$ (we set $e_{p+1}=e_{1}$ ).

- $\sigma\left(e_{i}\right)$ is the end edge of a reverse if $j=i-1$ (we set $e_{0}=e_{p}$ ).

In the following we write $\sigma_{1} \cdots \sigma_{p}$ for the pseudocycle $\sigma\left(e_{1}\right) \cdots \sigma\left(e_{p}\right)$.

Let $\Delta$ be a maximal tree of a normal graph $\Gamma$ on $q+1$ vertices. Then $\Delta$ has $q$ edges. Let $v_{1}, v_{2}$ be two vertices adjacent to the same edge $\sigma$ of $\Delta$. Write $\sigma\left(f_{1}\right)$ for $\sigma$. We define the circuit $\sigma\left(f_{1}\right) \cdots \sigma\left(f_{2 q}\right)$ as follows:

- if the vertex $v_{j+1}$ is not univalent, then $\sigma\left(f_{j+1}\right)$ is the first edge on the left of $\sigma\left(f_{j}\right)$ (we consider $\sigma\left(f_{j}\right)$ going from $v_{j}$ to $v_{j+1}$ ) and the vertex $v_{j+2}$ is the other vertex adjacent to $\sigma\left(f_{j+1}\right)$;

- if $v_{j+1}$ is univalent, then $\sigma\left(f_{j+1}\right)=\sigma\left(f_{j}\right)$ and $v_{j+2}=v_{j}$.

This way we come back to $v_{1}$ after passing twice through each edge of $\Delta$. Write $\delta_{v_{1}, v_{2}}(\Delta)$ for the word in $X_{\Gamma}$ corresponding to the circuit $\sigma\left(f_{1}\right) \cdots \sigma\left(f_{2 q}\right)$ (Figure 2). 


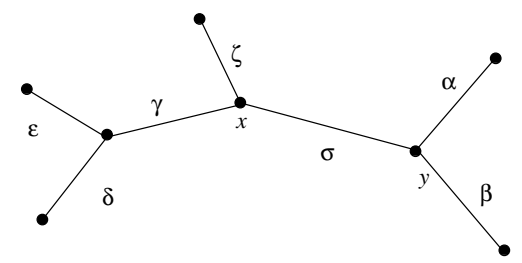

Fig. 2. $\delta_{x, y}(\Delta)=\sigma \alpha^{2} \beta^{2} \sigma \gamma \delta^{2} \varepsilon^{2} \gamma \zeta^{2}$ and $\delta_{y, x}(\Delta)=\sigma \gamma \delta^{2} \varepsilon^{2} \gamma \zeta^{2} \sigma \alpha^{2} \beta^{2}$

THEOREM 2.1. Let $\Gamma$ be a normal graph with $n$ vertices. The braid group $B_{n}\left(S^{2}\right)$ admits a presentation $\left\langle X_{\Gamma} \mid R_{\Gamma}\right\rangle$, where $X_{\Gamma}=\{\sigma \mid \sigma$ is an edge of $\Gamma\}$ and $R_{\Gamma}$ is the set of following relations:

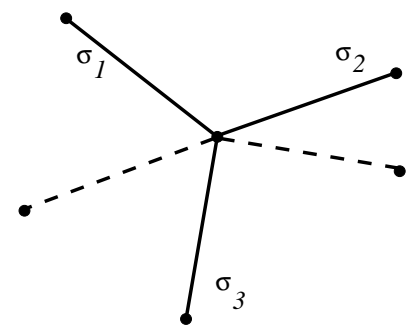

Fig. 3. Nodal relation
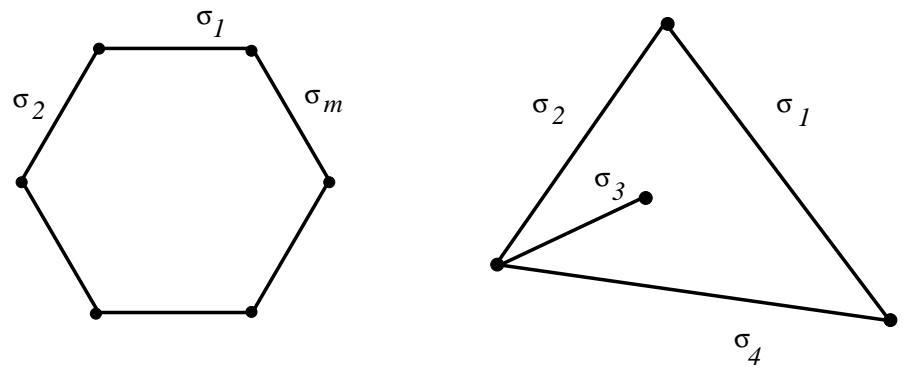

Fig. 4. Pseudocycle relation. On the left $\sigma_{1} \sigma_{2} \cdots \sigma_{m-1}=\sigma_{2} \cdots \sigma_{m}=\cdots=\sigma_{m} \cdots \sigma_{m-2}$; on the right $\sigma_{1} \sigma_{2} \sigma_{3}^{2}=\sigma_{2} \sigma_{3}^{2} \sigma_{4}=\sigma_{3}^{2} \sigma_{4} \sigma_{1}$ and $\sigma_{3} \sigma_{4} \sigma_{1} \sigma_{2}=\sigma_{4} \sigma_{1} \sigma_{2} \sigma_{3}$

- Disjointedness relations (DR): if $\sigma_{i}$ and $\sigma_{j}$ are disjoint, then $\sigma_{i} \sigma_{j}=$ $\sigma_{j} \sigma_{i}$

- Adjacency relations (AR): if $\sigma_{i}, \sigma_{j}$ have a common vertex, then $\sigma_{i} \sigma_{j} \sigma_{i}$ $=\sigma_{j} \sigma_{i} \sigma_{j}$

- Nodal relations (NR): if $\left\{\sigma_{1}, \sigma_{2}, \sigma_{3}\right\}$ have only one common vertex and they are clockwise oriented (Figure 3 ), then

$$
\sigma_{1} \sigma_{2} \sigma_{3} \sigma_{1}=\sigma_{2} \sigma_{3} \sigma_{1} \sigma_{2}
$$

- Pseudocycle relations (PR): if $\sigma_{1} \cdots \sigma_{m}$ is a pseudocycle and $\sigma_{1}$ is not 
the start edge nor is $\sigma_{m}$ the end edge of a reverse (Figure 4 ), then

$$
\sigma_{1} \sigma_{2} \cdots \sigma_{m-1}=\sigma_{2} \sigma_{3} \cdots \sigma_{m} .
$$

- Tree relations (TR): $\delta_{x, y}(\Delta)=1$ for every maximal tree $\Delta$ of $\Gamma$ and every ordered pair of vertices $x, y$ that are adjacent to the same edge $\sigma$ of $\Delta$.

REMARK 2.2. The statement of Theorem 2.1 is highly redundant. For instance one can show that a (TR) relation on a given maximal tree of $\Gamma$, together with (DR), (AR), (NR) and (PR), generate (TR) for any other maximal tree of $\Gamma$. Anyway, these presentations are symmetric and one can read off the relations from the geometry of $\Gamma$.

REMARK 2.3. Let $\gamma \subseteq \Gamma$ be a star (a graph which consists of several edges joined in one point). For any clockwise ordered subset $\left\{\sigma_{i_{1}}, \ldots\right.$, $\left.\sigma_{i_{j}} \mid j \geq 2\right\}$ of edges of $\gamma$ the following relation holds in the group $\left\langle X_{\Gamma} \mid R_{\Gamma}\right\rangle$ :

$$
\sigma_{i_{1}} \cdots \sigma_{i_{j}} \sigma_{i_{1}}=\sigma_{i_{j}} \sigma_{i_{1}} \cdots \sigma_{i_{j}} .
$$

2.2. Geometric interpretation of relations. It is geometrically evident that (AR) and (DR) hold in $B_{\Gamma}\left(S^{2}\right)$. Let $\Gamma$ contain a triangle $\sigma_{1}, \sigma_{2}, \tau$ as in Figure 8. The corresponding braids satisfy the relation $\tau=\sigma_{1} \sigma_{2} \sigma_{1}^{-1}$ and thus $\tau \sigma_{1}=\sigma_{1} \sigma_{2}$ in $B_{\Gamma}\left(S^{2}\right)$. The relation $\sigma_{1} \sigma_{2}=\sigma_{2} \tau$ follows from the braid relation $\sigma_{1} \sigma_{2} \sigma_{1}^{-1}=\sigma_{2}^{-1} \sigma_{1} \sigma_{2}$. Let $\sigma_{1}, \sigma_{2}, \sigma_{3}$ be arranged as in Figure 5 . We add three edges $\tau_{1}, \tau_{2}, \tau_{3}$. The nodal relation follows from the pseudocycle relations on the triangles $\tau_{1} \sigma_{2} \sigma_{3}, \tau_{2} \sigma_{1} \sigma_{3}$ and $\tau_{3} \sigma_{1} \sigma_{2}$. In fact, $\sigma_{1} \sigma_{2} \sigma_{3} \sigma_{1}=\sigma_{2} \tau_{3} \sigma_{3} \sigma_{1}=\sigma_{2} \sigma_{3} \tau_{3} \sigma_{1}=\sigma_{2} \sigma_{3} \sigma_{1} \sigma_{2}$. All other pseudocycle relations follow by induction on the length of the cycle.

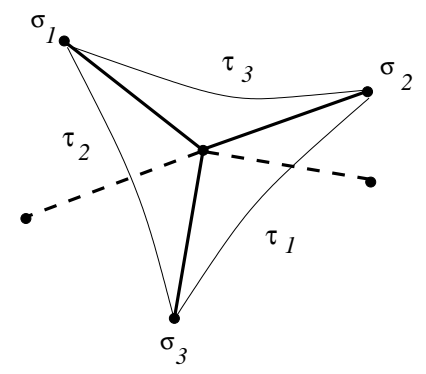

Fig. 5. Nodal relation holds in $B_{\Gamma}\left(S^{2}\right)$

Let $\Delta$ be a maximal tree of $\Gamma$. Let $\sigma$ be an edge of $\Delta$ and let $x, y$ be the two adjacent vertices. The element $\delta_{x, y}(\Delta)$ corresponds to a (pure) braid such that the braid obtained by removing the string starting from the vertex $x$ is isotopic to the trivial braid. This string goes around (with clockwise orientation) all other vertices (Figure 6 , left). The braid $\delta_{x, y}(\Delta)$ 
is isotopic to the trivial braid in $B_{\Gamma}\left(S^{2}\right)$ and so $\delta_{x, y}(\Delta)=1$ (Figure 6). Therefore the natural map $\phi_{\Gamma}:\left\langle X_{\Gamma} \mid R_{\Gamma}\right\rangle \rightarrow B_{\Gamma}\left(S^{2}\right)$ is a homomorphism.
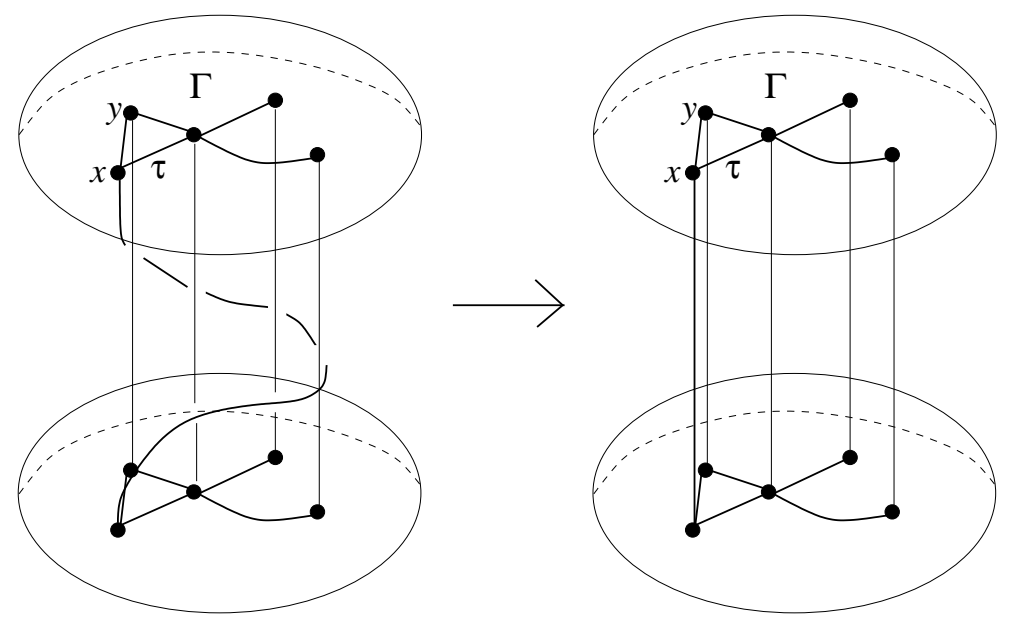

Fig. 6. The braid $\delta_{x, \sigma}(\Delta)$ associated to the tree $\Delta=\Gamma \backslash \tau$

3. Proof of Theorem 2.1. We need some preliminary lemmas.

Lemma 3.1. Let $\Gamma$ be a normal graph on the sphere and let $P$ be a pseudocycle of $\Gamma$. Let $R_{P}$ denote the set of pseudocycle relations associated to $P$. The group presentations $\left\langle X_{\Gamma} \mid R_{\Gamma}\right\rangle$ and $\left\langle X_{\Gamma} \mid R_{\Gamma} \backslash\left\{R_{P}\right\}\right\rangle$ are isomorphic.

Proof. We consider the sphere $S^{2}$ as the unit sphere in $\mathbb{R}^{3}$. We suppose that the disk bounded by $P$ contains the point $(0,0,-1)$. Then we enlarge the disk bounded by $P$ and we can also suppose that the graph $\Gamma$ is contained in the half-space $z>0$. We orthogonally project the graph on the $x y$ plane along the $z$-axis. Let $\sigma_{1}, \ldots, \sigma_{m}$ be the edges of the pseudocycle $P$ as in Figure 7. Since we consider the projection of $\Gamma$ on the $x y$-plane, the pseudocycle relations on $P$ will be clockwise oriented.
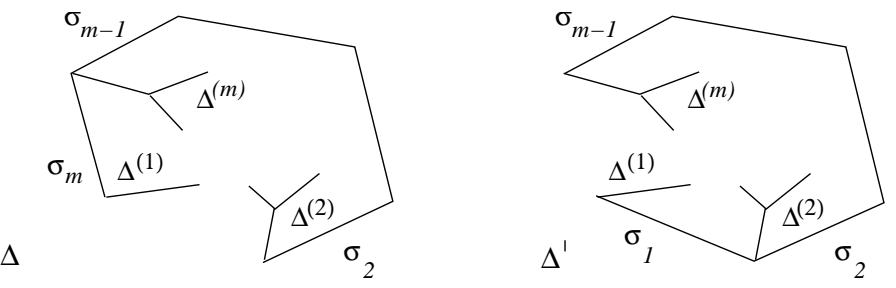

Fig. 7. The maximal trees $\Delta$ and $\Delta^{\prime}$ of $\Gamma$

Therefore, in order to prove the lemma we need to show that the relation $\sigma_{m} \cdots \sigma_{2}=\sigma_{m-1} \cdots \sigma_{1}$ holds in $\left\langle X_{\Gamma} \mid R_{\Gamma} \backslash\left\{R_{P}\right\}\right\rangle$. 
Consider two maximal trees $\Delta, \Delta^{\prime}$ of $\Gamma$ such that the tree $\Delta$ contains $\sigma_{2}, \ldots, \sigma_{m}$, the tree $\Delta^{\prime}$ contains $\sigma_{1}, \ldots, \sigma_{m-1}$, and $\Delta \cup\left\{\sigma_{1}\right\}=\Delta^{\prime} \cup\left\{\sigma_{m}\right\}$. The graph $\Delta \backslash\left\{\sigma_{2}, \ldots, \sigma_{m}\right\}=\Delta^{\prime} \backslash\left\{\sigma_{1}, \ldots, \sigma_{m-1}\right\}$ is a set of subtrees $\Delta^{(1)}, \Delta^{(2)}, \ldots, \Delta^{(m)}$ of $\Gamma$ (Figure 7 ). The (TR) relations on $\Delta$ and $\Delta^{\prime}$ yield

$$
\sigma_{m} \cdots \sigma_{2} \alpha_{2} \sigma_{2} \alpha_{3} \cdots \sigma_{m} \alpha_{1}=\sigma_{m-1} \cdots \sigma_{1} \alpha_{1} \sigma_{1} \alpha_{2} \cdots \sigma_{m-1} \alpha_{m},
$$

where $\alpha_{1}, \ldots, \alpha_{m}$ are subwords associated to the respective subtrees $\Delta^{(1)}, \ldots$ $\ldots, \Delta^{(m)}$. Let $\alpha_{1}=\zeta_{1} \cdots \zeta_{n-1} \zeta_{n} \zeta_{n+1} \cdots \zeta_{q}$, where $\zeta_{n-1}=\zeta_{n}$ and $\zeta_{i} \neq \zeta_{j}$, for $i, j \geq n$. We apply the $(\mathrm{PR})$ relation to the pseudocycle $\zeta_{n} \zeta_{n+1} \cdots \zeta_{q}$ $\sigma_{1} \alpha_{2} \cdots \sigma_{m} \zeta_{1} \cdots \zeta_{n-2} \zeta_{n-1}$, to get

$$
\zeta_{n} \zeta_{n+1} \cdots \zeta_{q} \sigma_{1} \alpha_{2} \cdots \sigma_{m} \zeta_{1} \cdots \zeta_{n-2}=\zeta_{n+1} \cdots \zeta_{q} \sigma_{1} \alpha_{2} \cdots \sigma_{m} \zeta_{1} \cdots \zeta_{n-2} \zeta_{n-1}
$$

and we derive

$$
\zeta_{q}^{-1} \cdots \zeta_{n+1}^{-1} \zeta_{n} \zeta_{n+1} \cdots \zeta_{q} \sigma_{1} \alpha_{2} \cdots \sigma_{m} \zeta_{1} \cdots \zeta_{n-2}=\sigma_{1} \alpha_{2} \cdots \sigma_{m} \zeta_{1} \cdots \zeta_{n-2} \zeta_{n-1} \text {. }
$$

Multiply by $\sigma_{1}^{-1}$ and apply (AR) to obtain

$\zeta_{n} \zeta_{n+1} \cdots \zeta_{q} \sigma_{1} \zeta_{q}^{-1} \cdots \zeta_{n+1}^{-1} \zeta_{n}^{-1} \alpha_{2} \cdots \sigma_{m} \zeta_{1} \cdots \zeta_{n-2}=\alpha_{2} \cdots \sigma_{m} \zeta_{1} \cdots \zeta_{n-2} \zeta_{n-1}$.

It follows that

$\alpha_{1} \sigma_{1} \alpha_{1}^{-1}$

$$
=\zeta_{1} \cdots \zeta_{n-1} \alpha_{2} \cdots \sigma_{m} \zeta_{1} \cdots \zeta_{n-2} \zeta_{n-1}\left(\zeta_{1} \cdots \zeta_{n-1} \alpha_{2} \cdots \sigma_{m} \zeta_{1} \cdots \zeta_{n-2}\right)^{-1} \text {. }
$$

From iterated applications of (AR) and (NR) it follows that

$$
\sigma_{m} \zeta_{1} \cdots \zeta_{n-2} \zeta_{n-1} \zeta_{n-2}^{-1} \cdots \zeta_{1}^{-1} \sigma_{m}^{-1}=\zeta_{n-1}^{-1} \zeta_{n-2}^{-1} \cdots \zeta_{1}^{-1} \sigma_{m} \zeta_{1} \cdots \zeta_{n-2} \zeta_{n-1} .
$$

Then

$$
\alpha_{1} \sigma_{1} \alpha_{1}^{-1}=\alpha_{2} \sigma_{2} \cdots \alpha_{m} \sigma_{m} \alpha_{m}^{-1} \cdots \sigma_{2}^{-1} \alpha_{2}^{-1}
$$

holds in $\left\langle X_{\Gamma} \mid R_{\Gamma} \backslash\left\{R_{P}\right\}\right\rangle$. The braids $\alpha_{1}$ and $\alpha_{m}^{-1} \cdots \sigma_{2}^{-1} \alpha_{2}^{-1}$ commute, and thus

$$
\alpha_{2} \sigma_{2} \cdots \alpha_{m} \sigma_{m} \alpha_{1}=\alpha_{1} \sigma_{1} \alpha_{2} \cdots \alpha_{m} .
$$

From equation (1), it follows that

$$
\sigma_{m} \cdots \sigma_{2}=\sigma_{m-1} \cdots \sigma_{1}
$$

The following lemma generalises a result on classical braids and planar graphs proved in [30] (Lemmas 2 and 3) to braids on the sphere. The lemma states that for any graph $\Gamma^{\prime}$ obtained from a given $\Gamma$ on $S^{2}$ by removing or adding "triangles" the groups $\left\langle X_{\Gamma^{\prime}} \mid R_{\Gamma^{\prime}}\right\rangle$ and $\left\langle X_{\Gamma} \mid R_{\Gamma}\right\rangle$ defined in Theorem 2.1 are isomorphic.

LEMma 3.2. Let $\sigma_{1}, \sigma_{2}$ be two adjacent edges of $\Gamma$, not contained in any pseudocycle. Let $\Gamma^{\prime}=\Gamma \cup \tau$ be the graph obtained by adding an edge $\tau$ to $\Gamma$ to form an anticlockwise triangle $\tau \sigma_{1} \sigma_{2}$ (Figure 8). Then $\left\langle X_{\Gamma} \mid R_{\Gamma}\right\rangle$ is a group presentation for $B_{\Gamma}\left(S^{2}\right)$ if and only if $\left\langle X_{\Gamma^{\prime}} \mid R_{\Gamma^{\prime}}\right\rangle$ is a group presentation for $B_{\Gamma^{\prime}}\left(S^{2}\right)$. 


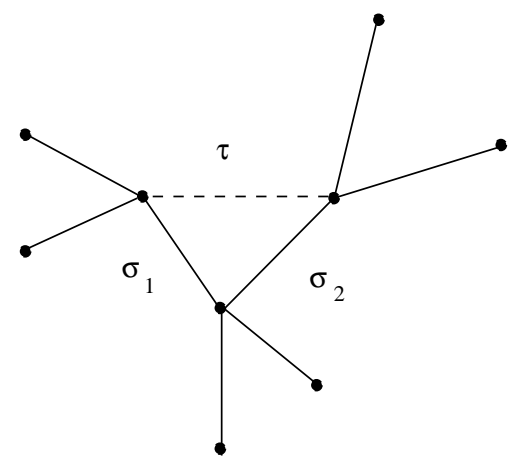

Fig. 8. Adding or removing a triangle

Proof. Note that by the Tietze transformation $\left\langle X_{\Gamma^{\prime}} \mid R_{\Gamma}, \tau=\sigma_{1} \sigma_{2} \sigma_{1}^{-1}\right\rangle$ is isomorphic to $\left\langle X_{\Gamma} \mid R_{\Gamma}\right\rangle$. Hence in order to prove that $\left\langle X_{\Gamma^{\prime}} \mid R_{\Gamma^{\prime}}\right\rangle$ is a group presentation for $B_{\Gamma^{\prime}}\left(S^{2}\right)$ if and only if $\left\langle X_{\Gamma^{\prime}} \mid R_{\Gamma^{\prime}}\right\rangle$ is a group presentation for $B_{\Gamma}\left(S^{2}\right)$, it is sufficient to prove that the groups $\left\langle X_{\Gamma^{\prime}} \mid R_{\Gamma^{\prime}}\right\rangle$ and $\left\langle X_{\Gamma^{\prime}} \mid R_{\Gamma}, \tau=\sigma_{1} \sigma_{2} \sigma_{1}^{-1}\right\rangle$ are isomorphic. Since $\left\{R_{\Gamma}, \tau=\sigma_{1} \sigma_{2} \sigma_{1}^{-1}\right\} \subset R_{\Gamma^{\prime}}$, we just need to show that the relations $R_{\Gamma^{\prime}}$ are satisfied in the group $\left\langle X_{\Gamma^{\prime}} \mid R_{\Gamma}, \tau=\sigma_{1} \sigma_{2} \sigma_{1}^{-1}\right\rangle$. Sergiescu showed (Lemma 1.3 in [30]) that (DR), (AR) and (NR) for $\Gamma^{\prime}$ are consequences of (DR), (AR) and (NR) for $\Gamma$ and of the relation $\tau=\sigma_{1} \sigma_{2} \sigma_{1}^{-1}$. The edge $\tau$ belongs to the pseudocycle $\tau \sigma_{1} \sigma_{2}$. The corresponding pseudocycle relations derive from $\tau=\sigma_{1} \sigma_{2} \sigma_{1}^{-1}$ and $\sigma_{1} \sigma_{2} \sigma_{1}=\sigma_{2} \sigma_{1} \sigma_{2}$. We prove that the relation $\delta_{x, y}(\Delta)=1$ is a consequence of the set of relations $R_{\Gamma} \cup\left\{\tau=\sigma_{1} \sigma_{2} \sigma_{1}^{-1}\right\}$, for every maximal tree $\Delta$ of $\Gamma^{\prime}$ and every ordered pair of vertices $x, y$ that are adjacent to the same edge $\sigma$ of $\Delta$. If $\tau \notin \Delta$ the claim follows. Suppose that $\tau \in \Delta$. We have two cases (we refer to Figure 9):

(1) $\delta_{x, y}(\Delta)=\alpha_{0} \tau \alpha_{1} \sigma_{2} \alpha_{2} \sigma_{2} \tau \alpha_{3}$, where the $\alpha_{i}$ are the subwords obtained by following the rest of $\Delta$;

(2) $\delta_{x, y}(\Delta)=\beta_{0} \sigma_{1} \beta_{1} \tau \beta_{2} \tau \sigma_{1} \beta_{3}$, where the $\beta_{i}$ are the subwords obtained by following the rest of $\Delta$.
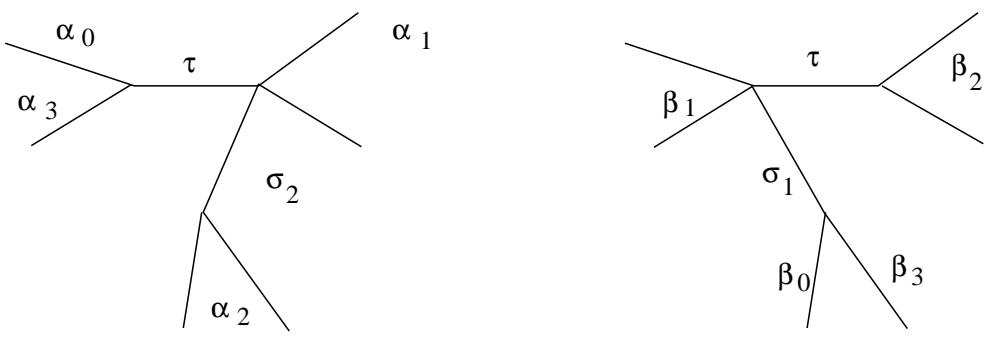

Fig. 9. Tree relations for $\Gamma^{\prime}=\Gamma \cup \tau$ are generated by the set $R_{\Gamma} \cup\left\{\tau=\sigma_{1} \sigma_{2} \sigma_{1}^{-1}\right\}$ 
Replace $\tau$ by $\sigma_{1} \sigma_{2} \sigma_{1}^{-1}$. In the first case, write $\alpha_{2}=\sigma_{3} \zeta_{3} \sigma_{3} \cdots \sigma_{p} \zeta_{p} \sigma_{p}$, where $\sigma_{k}$ for $k=1, \ldots, p$ corresponds to an edge of $\Gamma$ adjacent to $\sigma_{1}$ and $\sigma_{2}$, and $\zeta_{k}$ for $k=1, \ldots, p$ corresponds to a tree disjoint from $\sigma_{1}$ and $\sigma_{2}$. The elements $\sigma_{k}(k=1, \ldots, p)$ and $\sigma_{1} \sigma_{2} \sigma_{1}^{-1}$ commute, so that

$$
\sigma_{k} \sigma_{1} \sigma_{2} \sigma_{1}^{-1}=\sigma_{2}^{-1} \sigma_{1} \sigma_{2} \sigma_{k}=\sigma_{1} \sigma_{2} \sigma_{1}^{-1} \sigma_{k}
$$

One derives that $\left[\alpha_{2}, \sigma_{1} \sigma_{2} \sigma_{1}^{-1}\right]=1$. From $\sigma_{1} \alpha_{1}=\alpha_{1} \sigma_{1}$ it follows that

$$
\begin{aligned}
& \alpha_{0} \sigma_{1} \sigma_{2} \sigma_{1}^{-1} \alpha_{1} \sigma_{2} \alpha_{2} \sigma_{2} \sigma_{1} \sigma_{2} \sigma_{1}^{-1} \alpha_{3}=\alpha_{0} \sigma_{1} \sigma_{2} \alpha_{1} \sigma_{1}^{-1} \sigma_{2} \alpha_{2} \sigma_{1} \sigma_{2} \sigma_{1}^{-1} \sigma_{1} \alpha_{3} \\
& =\alpha_{0} \sigma_{1} \sigma_{2} \alpha_{1} \sigma_{1}^{-1} \sigma_{2} \sigma_{1} \sigma_{2} \sigma_{1}^{-1} \alpha_{2} \sigma_{1} \alpha_{3}=\alpha_{0} \sigma_{1} \sigma_{2} \alpha_{1} \sigma_{2} \alpha_{2} \sigma_{1} \alpha_{3} .
\end{aligned}
$$

In the second case, from (DR) it follows that $\sigma_{1} \beta_{2}=\beta_{2} \sigma_{1}$ and then

$$
\begin{aligned}
\beta_{0} \sigma_{1} \beta_{1} \sigma_{1} \sigma_{2} \sigma_{1}^{-1} \beta_{2} \sigma_{1} \sigma_{2} \sigma_{1}^{-1} \sigma_{1} \beta_{3} & =\beta_{0} \sigma_{1} \beta_{1} \sigma_{1} \sigma_{2} \sigma_{1}^{-1} \beta_{2} \sigma_{1} \sigma_{2} \beta_{3} \\
& =\beta_{0} \sigma_{1} \beta_{1} \sigma_{1} \sigma_{2} \beta_{2} \sigma_{2} \beta_{3} .
\end{aligned}
$$

One deduces that the relation $\delta_{x, y}(\Delta)=1$ holds in $\left\langle X_{\Gamma^{\prime}} \mid R_{\Gamma}, \tau=\sigma_{1} \sigma_{2} \sigma_{1}^{-1}\right\rangle$. We remark that $\tau$ also belongs to the pseudocycle $P$ bounding the other connected component of $S^{2} \backslash \Gamma$. According to Lemma 3.1 we can suppose that the pseudocycle relations associated to $P$ are redundant and thus $\left\langle X_{\Gamma^{\prime}} \mid R_{\Gamma^{\prime}}\right\rangle$ is isomorphic to $\left\langle X_{\Gamma^{\prime}} \mid R_{\Gamma}, \tau=\sigma_{1} \sigma_{2} \sigma_{1}^{-1}\right\rangle$.

\subsection{Inductive steps}

Definition 3.3. A node is a vertex of valence greater than two. We define the valence of $\Gamma, v(\Gamma)$, to be the sum of the valences of all nodes of $\Gamma$.

In order to prove Theorem 2.1, we proceed by induction on the number of connected components of $S^{2} \backslash \Gamma$.

(i) Let $\Gamma$ be a tree. We recall that the braid group of the sphere is a quotient of the braid group of the disk.

THEOREM $3.4([18,37])$. The group $B_{n}\left(S^{2}\right)$ admits the following presentation:

- Generators: $\sigma_{1}, \ldots, \sigma_{n-1}$;

- Relations:

$$
\begin{aligned}
\sigma_{i} \sigma_{i+1} \sigma_{i} & =\sigma_{i+1} \sigma_{i} \sigma_{i+1} \\
\sigma_{i} \sigma_{j} & =\sigma_{j} \sigma_{i} \quad \text { for }|i-j| \geq 2 \\
\sigma_{1} \sigma_{2} \cdots \sigma_{n-1}^{2} \cdots \sigma_{2} \sigma_{1} & =1 .
\end{aligned}
$$

It follows that the assertion of Theorem 2.1 holds when $\Gamma$ is a straight line and $v(\Gamma)=0$. Suppose that it holds for all trees with valence less than $q>0$ and let $\Gamma$ be a tree such that $v(\Gamma)=q$. Let $v_{0}$ be a univalent vertex of $\Gamma$. We follow the tree $\Gamma$ starting from $v_{0}$ and turning right at each node. 
Let $v_{1}$ be the vertex preceding the first node. Let $v_{2}$ be the first univalent vertex after $v_{0}$ (see Figure 10).

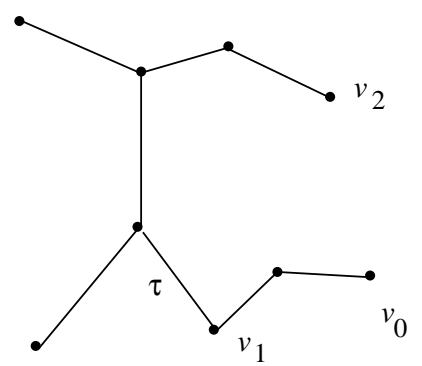

$\Gamma$

Fig. 10. We suppose $\Gamma$ embedded in the sphere

We replace the edge $\tau$ between $v_{1}$ and the first node with an edge $\tau_{1}$ joining $v_{1}$ to the vertex $v_{2}$ (see Figure 11). The graph $\Gamma_{1}$ so obtained is such that $v\left(\Gamma_{1}\right)<v(\Gamma)$, and thus the assertion of Theorem 2.1 holds for $\Gamma_{1}$.

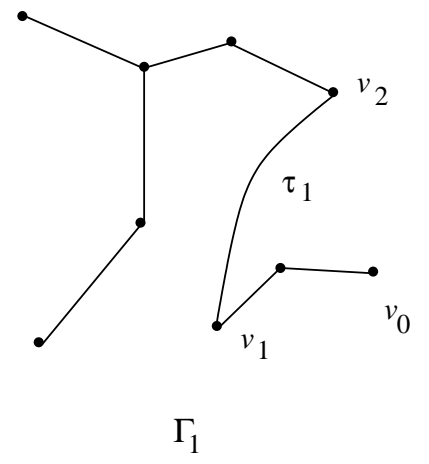

Fig. 11. Replacing the edge $\tau$ with $\tau_{1}$

From Lemma 3.2 it follows that this assertion is also satisfied for the graph $\Gamma_{2}$ obtained by adding an edge $\tau_{2}$ between $v_{1}$ and the other vertex adjacent to $v_{2}$ (see Figure 12).

From Lemma 3.2 one deduces that the conclusion of Theorem 2.1 holds for the graph $\Gamma_{3}=\Gamma_{2} \backslash\left\{\tau_{1}\right\}$. Iterating the process we derive that it holds for the initial tree $\Gamma$.

(ii) Suppose that the conclusion of Theorem 2.1 holds when the number of connected components of $S^{2} \backslash \Gamma$ is less than $p>1$. Let $\Gamma$ be a normal graph such that $S^{2} \backslash \Gamma$ has $p$ connected components. We remove an edge $\sigma_{1}$ which bounds two pseudocycles $\sigma_{1}, \sigma_{2}, \ldots, \sigma_{n}$ and $\sigma_{1}, \tau_{2}, \ldots, \tau_{m}$ of $\Gamma$. We encounter two cases. 


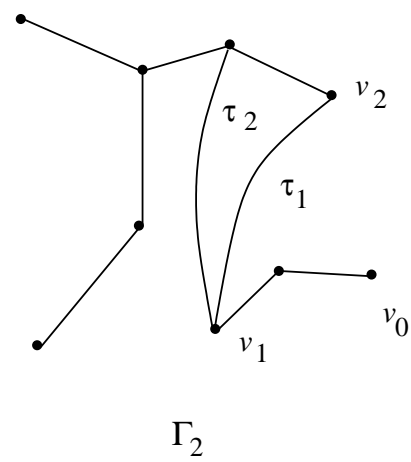

Fig. 12. Adding and removing triangulations

(1) $\sigma_{n}$ is not the end edge of a reverse. From the induction hypothesis and Tietze transformation, we deduce that $\left\langle X_{\Gamma}\right| R_{\Gamma \backslash\left\{\sigma_{1}\right\}}, \sigma_{1} \cdots \sigma_{n-1}=$ $\left.\sigma_{2} \cdots \sigma_{n}\right\rangle$ is a group presentation for $B_{\Gamma}\left(S^{2}\right)$. As we remarked in Section 2.2, the set $R_{\Gamma}$ is a system of relations for $B_{n}\left(S^{2}\right)$. Since $\left\{R_{\Gamma \backslash\left\{\sigma_{1}\right\}}, \sigma_{1} \cdots \sigma_{n-1}=\right.$ $\left.\sigma_{2} \cdots \sigma_{n}\right\}$ is a complete system of relations for $B_{n}\left(S^{2}\right)$ and it is a subset of $R_{\Gamma}$, we conclude that $\left\langle X_{\Gamma} \mid R_{\Gamma}\right\rangle$ is a group presentation for $B_{\Gamma}\left(S^{2}\right)$.

(2) Suppose that $\sigma_{n}$ is the end edge of a reverse. If there exists $l<n$ such that $\sigma_{l}=\sigma_{l+1}$ and $\sigma_{i} \neq \sigma_{j}$ for $l+1 \leq i<j \leq n$, then the relation

$$
\sigma_{l+1} \sigma_{l+2} \cdots \sigma_{n} \sigma_{1} \sigma_{2} \ldots \sigma_{l-1}=\sigma_{l+2} \cdots \sigma_{n} \sigma_{1} \sigma_{2} \cdots \sigma_{l-1} \sigma_{l}
$$

holds in $\left\langle X_{\Gamma} \mid R_{\Gamma}\right\rangle$. Multiplying by $\sigma_{1}^{-1} \sigma_{n}^{-1} \cdots \sigma_{l+2}^{-1}$ and applying (AR) we obtain

$$
\begin{aligned}
\sigma_{2} \ldots \sigma_{l-1} \sigma_{l} & =\sigma_{1}^{-1} \sigma_{n}^{-1} \cdots \sigma_{l+2}^{-1} \sigma_{l+1} \sigma_{l+2} \cdots \sigma_{n} \sigma_{1} \sigma_{2} \cdots \sigma_{l-1} \\
& =\sigma_{l+1} \sigma_{l+2} \cdots \sigma_{n} \sigma_{1} \sigma_{n}^{-1} \cdots \sigma_{l+2}^{-1} \sigma_{l+1}^{-1} \sigma_{2} \cdots \sigma_{l-1},
\end{aligned}
$$

which yields

$$
\sigma_{1}=\sigma_{n}^{-1} \cdots \sigma_{l+1}^{-1} \sigma_{2} \cdots \sigma_{l-1} \sigma_{l} \sigma_{l-1}^{-1} \cdots \sigma_{2}^{-1} \sigma_{l+1} \cdots \sigma_{n} .
$$

Otherwise if $\sigma_{l}=\sigma_{l+1}$ only for $l=n-1$, then the relation

$$
\sigma_{n} \sigma_{1} \sigma_{2} \cdots \sigma_{n-2}=\sigma_{1} \sigma_{2} \cdots \sigma_{n-2} \sigma_{n-1}
$$

holds in $\left\langle X_{\Gamma} \mid R_{\Gamma}\right\rangle$. Multiplying by $\sigma_{1}^{-1}$ and applying (AR) we obtain

$$
\sigma_{2} \cdots \sigma_{n-2} \sigma_{n-1}=\sigma_{1}^{-1} \sigma_{n} \sigma_{1} \sigma_{2} \cdots \sigma_{n-2}=\sigma_{n} \sigma_{1} \sigma_{n}^{-1} \sigma_{2} \cdots \sigma_{n-2},
$$

which yields

$$
\sigma_{1}=\sigma_{n}^{-1} \sigma_{2} \cdots \sigma_{n-2} \sigma_{n-1} \sigma_{n-2}^{-1} \cdots \sigma_{2}^{-1} \sigma_{n}
$$

This concludes the proof.

3.2. Automorphisms and isometries. Let $F$ be a subset of $\mathbb{R}^{2}$ (respectively of $S^{2}$ ). The symmetry group of $F, \Sigma(F)$, is the set of congruent transformations of $\mathbb{R}^{2}$ (respectively of $S^{2}$ ) that leave $F$ invariant. We denote by 
$\Sigma(F)^{+}$the subgroup of $\Sigma(F)$ generated by rotations. The symmetry group $\Sigma(F)$ is discrete if the set $\{\phi(P) \mid \phi \in \Sigma(F)\}$ is discrete for any point $P \in F$.

Corollary 3.5. Every finite subgroup $H$ of $O(3)$ is isomorphic to a subgroup of $\operatorname{Aut}\left(B_{n}\left(S^{2}\right)\right)$ for some $n$.

Proof. Let $\Gamma$ be a normal graph on the sphere such that $\Sigma(\Gamma)=H$ (see for instance [14]). Since relations of $B_{\Gamma}\left(S^{2}\right)$ are preserved by rotations we associate to every rotation $\varrho \in H$ the corresponding automorphism $\bar{\varrho}$ of $B_{\Gamma}\left(S^{2}\right)$. To every reflection $\chi \in H$ we associate the morphism $\bar{\chi}$ that moves the generator $\sigma$ of $B_{\Gamma}\left(S^{2}\right)$ to the braid $\chi(\sigma)^{-1}$. This map is an automorphism of $B_{\Gamma}\left(S^{2}\right)$. The subgroup $K$ of $\operatorname{Aut}\left(B_{\Gamma}\left(S^{2}\right)\right)$ generated by the set $\{\bar{g} \mid g$ a generator of $H$ \} is isomorphic to $H$.

4. Braids on the annulus. The braid groups are included in the series of so-called "generalised braid groups" defined by Brieskorn [10]. They were called Artin groups in [11], and are defined as the groups with generators $\left\{s_{i} \mid i \in I\right\}$ and relations

$$
\operatorname{prod}\left(m_{i, j}, s_{i}, s_{j}\right)=\operatorname{prod}\left(m_{j, i}, s_{j}, s_{i}\right) .
$$

We use here the notation of P. Deligne [15]: $\operatorname{prod}(m, x, y)$ denotes the product $x y x y \ldots$ ( $m$ factors $)$. From this presentation we obtain the presentation of the corresponding reflection group $W[12]$ by adding the relation $s_{i}^{2}=e$ for any $i \in I$.

Let us consider the $n$th Artin group of type $\mathcal{B}$. This group is given by the following presentation:

- Generators: $\tau, \sigma_{1}, \ldots, \sigma_{n-1}$;

- Relations:

$$
\begin{aligned}
\sigma_{i} \sigma_{i+1} \sigma_{i} & =\sigma_{i+1} \sigma_{i} \sigma_{i+1} & & (i=1, \ldots, n-2) ; \\
\sigma_{i} \sigma_{j} & =\sigma_{j} \sigma_{i} & & (|i-j| \geq 2) ; \\
\tau \sigma_{j} & =\sigma_{j} \tau & & (j \neq 1) ; \\
\tau \sigma_{1} \tau \sigma_{j} & =\sigma_{1} \tau \sigma_{j} \tau . & &
\end{aligned}
$$

This group can be identified with the fundamental group of the configuration space of distinct points on the plane with one point deleted [32], which means the same as the braid group on $n$ strands on the annulus, $B_{n}$ (Ann). A geometric interpretation of the generators $\tau, \sigma_{1}, \ldots, \sigma_{n-1}$ is given in Figure 13.

We recall that the group $B_{n}(\mathrm{Ann})$ embeds in the braid group $B_{n+1}$. More precisely, it is isomorphic to the subgroup of braids on $n+1$ strands whose first strand is isotopic to the trivial one. 


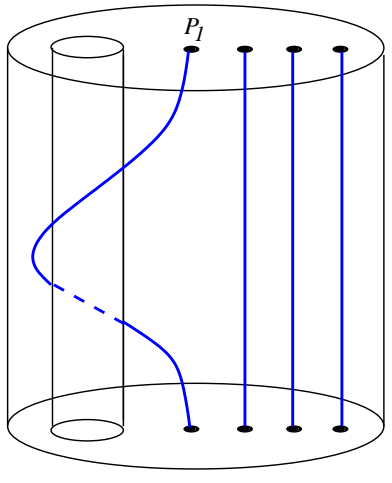

$\tau$

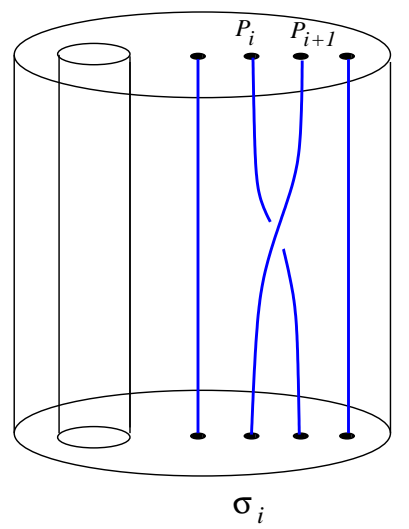

Fig. 13. Geometric interpretation of the generators $\tau, \sigma_{1}, \ldots, \sigma_{n-1}$ of $B_{n}(\mathrm{Ann})$

In the following we consider a normal (connected, finite and without loops or intersections) planar graph $\Gamma$ such that there exists a distinguished vertex $v$ with the property that the graph $\Gamma$ minus the vertex $v$ and all the edges adjacent to $v$ is still connected. We label by $\left\{\tau_{1}, \ldots, \tau_{m}\right\}$ the edges adjacent to $v$ and by $\left\{\sigma_{1}, \ldots, \sigma_{p}\right\}$ the other ones. We call $\Gamma$ a 1-punctured graph.

THEOREM 4.1. Let $\Gamma$ be a 1-punctured graph with $n+1$ vertices. The braid group $B_{n}(\mathrm{Ann})$ admits the presentation $\left\langle X_{\Gamma} \mid R_{\Gamma}\right\rangle$, where $X_{\Gamma}=$ $\left\{\sigma_{a}, \tau_{b} \mid a\right.$ is an edge of $\Gamma$ not adjacent to the distinguished vertex $v$ and $b$ is an edge adjacent to $v\}$ and $R_{\Gamma}$ is the set of the following relations:

- Disjointedness relations (DR): if the edges a and $c$ (respectively $b$ and c) are disjoint, then $\sigma_{a} \sigma_{c}=\sigma_{c} \sigma_{a}$ (respectively $\left.\tau_{b} \sigma_{c}=\sigma_{c} \tau_{b}\right)$;

- Adjacency relations (AR): if the edges a and $c$ (respectively $b$ and $c$ ) have a common vertex, then $\sigma_{a} \sigma_{c} \sigma_{a}=\sigma_{c} \sigma_{a} \sigma_{c}\left(\tau_{b} \sigma_{c} \tau_{b} \sigma_{c}=\sigma_{c} \tau_{b} \sigma_{c} \tau_{b}\right)$;

- Nodal relations (NR): Let $a, b, c$ be three edges that have only one common vertex and are clockwise ordered. If the edges $a, b, c$ are not adjacent to $v$, then

$$
\sigma_{a} \sigma_{b} \sigma_{c} \sigma_{a}=\sigma_{b} \sigma_{c} \sigma_{a} \sigma_{b}
$$

if the edges $a, c$ are not adjacent to $v$ and $b$ is adjacent to $v$, then

$$
\sigma_{a} \sigma_{b} \tau_{c} \sigma_{a}=\sigma_{b} \tau_{c} \sigma_{a} \sigma_{b}, \quad \tau_{b} \sigma_{c} \sigma_{a} \tau_{b} \sigma_{c}=\sigma_{a} \tau_{b} \sigma_{c} \sigma_{a} \tau_{b}
$$

- Pseudocycle relations (PR): if the edges $a_{1}, \ldots, a_{m}$ form a pseudocycle, $a_{1}$ is not the start edge nor is $a_{m}$ the end edge of a reverse, and no $a_{i}$ is adjacent to $v$, then

$$
\sigma_{a_{1}} \sigma_{a_{2}} \cdots \sigma_{a_{m-1}}=\sigma_{a_{2}} \sigma_{a_{3}} \cdots \sigma_{a_{m}}
$$


If $a_{1}, a_{m}$ are adjacent to $v$, then

$$
\tau_{a_{1}} \sigma_{a_{2}} \cdots \sigma_{a_{m-1}}=\sigma_{a_{2}} \sigma_{a_{3}} \cdots \tau_{a_{m}} .
$$

REMARK 4.2. As in Theorem 2.1, the nodal relation (NR) also implies the equality

$$
\sigma_{a} \sigma_{b} \sigma_{c} \sigma_{a}=\sigma_{b} \sigma_{c} \sigma_{a} \sigma_{b}=\sigma_{c} \sigma_{a} \sigma_{b} \sigma_{c} .
$$

The geometric interpretation of the generators is the following. The distinguished vertex corresponds to the deleted point of $\mathbb{R}$. To any edge $a$ not adjacent to $v$ we associate the corresponding positive half-twist as in Figure 1 . To any edge $b$ adjacent to $v$ we associate the braid $\tau_{b}$ as in Figure 14 .
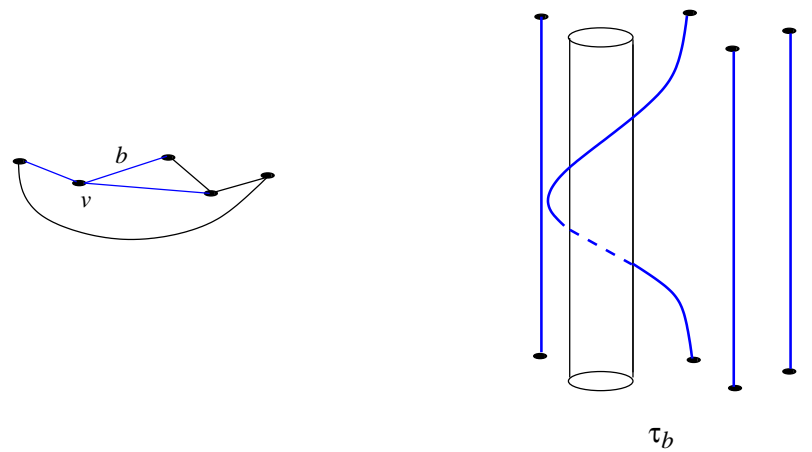

Fig. 14. Geometric interpretation of $\tau_{b}$

We show the geometric interpretation of the relation $\tau_{b} \sigma_{c} \sigma_{a} \tau_{b} \sigma_{c}=$ $\sigma_{a} \tau_{b} \sigma_{c} \sigma_{a} \tau_{b}$. The other ones can be easily verified as in Section 2.2. We add two edges $d$ and $e$, with their corresponding braids $\tau_{d}$ and $\tau_{e}$ as in Figure 15. The braid $\tau_{d}$ is equivalent to $\sigma_{c}^{-1} \tau_{b} \sigma_{c}$, and $\tau_{e}$ is equivalent to $\sigma_{a} \tau_{b} \sigma_{a}^{-1}$. Then $\sigma_{c}^{-1} \tau_{b} \sigma_{c}$ and $\sigma_{a}$ commute, as do $\sigma_{a} \tau_{b} \sigma_{a}^{-1}$ and $\sigma_{c}$. So we have the following equalities, which can be easily verified on corresponding braids:

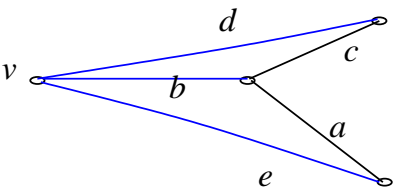

Fig. 15. Nodal relation $\tau_{b} \sigma_{c} \sigma_{a} \tau_{b} \sigma_{c}=\sigma_{a} \tau_{b} \sigma_{c} \sigma_{a} \tau_{b}$ holds in $B_{\Gamma}($ Ann $)$

$$
\begin{aligned}
& \tau_{b} \sigma_{c} \sigma_{a} \tau_{b} \sigma_{c}=\sigma_{c} \sigma_{c}^{-1} \tau_{b} \sigma_{c} \sigma_{a} \tau_{b} \sigma_{c}=\sigma_{c} \sigma_{a} \sigma_{c}^{-1} \tau_{b} \sigma_{c} \tau_{b} \sigma_{c} \\
& \quad=\sigma_{c} \sigma_{a} \sigma_{c}^{-1} \sigma_{c} \tau_{b} \sigma_{c} \tau_{b}=\sigma_{c} \sigma_{a} \tau_{b} \sigma_{a}^{-1} \sigma_{a} \sigma_{c} \tau_{b}=\sigma_{a} \tau_{b} \sigma_{a}^{-1} \sigma_{c} \sigma_{a} \sigma_{c} \tau_{b}=\sigma_{a} \tau_{b} \sigma_{c} \sigma_{a} \tau_{b} .
\end{aligned}
$$

A proof of Theorem 4.1 is given in [34]. The steps of the proof are the same as for Theorem 2.1. 
COROLlARY 4.3. The automorphism group of $B_{n}(\mathrm{Ann})$ contains a group isomorphic to the dihedral group $D_{n-1}$.

Proof. One can associate to the graph given in Figure 16 a presentation for $B_{n}(\mathrm{Ann})$. The same argument used in Corollary 3.5 concludes the proof.

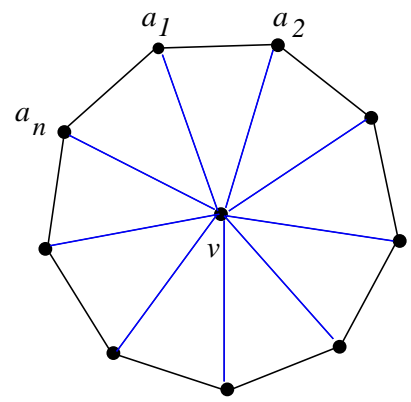

Fig. 16. A graph associated to $B_{n}(\mathrm{Ann})$

We remark that one can generalise this approach to braid groups on a planar surface, i.e. a surface of genus 0 with $l>1$ boundary components. In such a case one considers a normal planar graph with $k(=l-1)$ distinguished vertices $v_{1}, \ldots, v_{k}$ such that there are no edges connecting distinguished vertices and the graph $\Gamma$ minus the vertices $v_{1}, \ldots, v_{k}$ and all the edges adjacent to $v_{1}, \ldots, v_{k}$ is still connected. We label by $\left\{\tau_{1, j}, \ldots, \tau_{m, j}\right\}$ the edges adjacent to $v_{j}$ and by $\left\{\sigma_{1}, \ldots, \sigma_{p}\right\}$ the edges disjoint from $\left\{v_{1}, \ldots, v_{k}\right\}$. We say that $\Gamma$ is a $k$-punctured graph. As in Theorem 4.1 one can associate to any $k$-punctured graph $\Gamma$ on $n$ vertices a set of generators for the braid group on $n$ strands on a surface of genus 0 with $k+1$ boundary components, with the above geometrical interpretation of generators.

5. Singular braids and graphs. Singular braids have been introduced in $[1,8]$ as a generalisation of classical braids, by allowing strands to intersect in finitely many double points.

The set of singular braids on $n$ strands $S B_{n}$, up to isotopy, forms a monoid. To the standard generator $\sigma_{j}$ of the braid group $B_{n}$ one can associate the singular braid $x_{j}$ obtained by replacing the positive crossing of the $j$ th and $(j+1)$ th strands by a singular point.

Baez and Birman provided a monoid presentation for $S B_{n}$. The generators are $\sigma_{1}, \ldots \sigma_{n-1}$, their inverses $\sigma_{1}^{-1}, \ldots \sigma_{n-1}^{-1}$ and the singular braids $x_{1}, \ldots, x_{n-1}$. To the usual braid relations (and the invertibility of $\sigma_{j}$ ) one 
has to add the following relations:

$$
\begin{aligned}
x_{i} \sigma_{j} \sigma_{i} & =\sigma_{j} \sigma_{i} x_{j} & & \text { for }|i-j|=1 ; \\
x_{i} x_{j} & =x_{j} x_{j} & & \text { for }|i-j| \geq 2 ; \\
x_{i} \sigma_{j} & =\sigma_{j} x_{i} & & \text { for }|i-j| \geq 2 ; \\
x_{i} \sigma_{i} & =\sigma_{i} x_{i} & & \text { for } i=1, \ldots, n-1 .
\end{aligned}
$$

Singular braids are related to the Vassiliev-Goussarov theory of finite type invariants. The Birman-Ko-Lee presentation for the monoid $S B_{n}$ was obtained in [33]. As in the case of classical braids, one can extend the group $B_{n}(\Sigma)$ to the monoid $S B_{n}(\Sigma)$ of singular braids on $n$ strands on the surface $\Sigma$. This monoid has been introduced in [22], in order to define finite type invariants for surface braids. Presentations for this monoid are given in [2] and [21].

In this section we provide presentations by graphs for the monoid $S B_{n}$ and for the monoid $S B_{n}(\mathrm{Ann})$ of singular braids on $n$ strands of the annulus.

Let $\Gamma$ be a normal planar graph. We associate to any edge $a$ three singular braids: $\sigma_{a}$ will denote the positive half-twist associated to $a$ (as in Figure 1), $\sigma_{a}^{-1}$ will denote the corresponding negative half-twist, and $x_{a}$ the corresponding singular crossing.

THEOREM 5.1. Let $\Gamma$ be a normal planar graph with $n$ vertices. The singular braid monoid $S B_{n}$ has the presentation $\left\langle X_{\Gamma}, R_{\Gamma}\right\rangle$ where $X_{\Gamma}=$ $\left\{\sigma_{a}, \sigma_{a}^{-1}, x_{a} \mid a\right.$ is an edge of $\left.\Gamma\right\}$ and $R_{\Gamma}$ is formed by the following six types of relations:

- Disjointedness: if the edges $a$ and $b$ are disjoint, then

$$
\sigma_{a} \sigma_{b}=\sigma_{b} \sigma_{a}, \quad x_{a} x_{b}=x_{b} x_{a}, \quad \sigma_{a} x_{b}=x_{b} \sigma_{a} ;
$$

- Commutativity:

$$
\sigma_{a} x_{a}=x_{a} \sigma_{a}
$$

- Invertibility:

$$
\sigma_{a} \sigma_{a}^{-1}=\sigma_{a}^{-1} \sigma_{a}=1
$$

- Adjacency: if the edges a and $b$ have a common vertex, then

$$
\sigma_{a} \sigma_{b} \sigma_{a}=\sigma_{b} \sigma_{a} \sigma_{b}, \quad x_{a} \sigma_{b} \sigma_{a}=\sigma_{b} \sigma_{a} x_{b}
$$

- Nodal: if the edges $a, b$ and $c$ have a common vertex and are placed clockwise, then

$$
\begin{gathered}
\sigma_{a} \sigma_{b} \sigma_{c} \sigma_{a}=\sigma_{b} \sigma_{c} \sigma_{a} \sigma_{b}=\sigma_{c} \sigma_{a} \sigma_{b} \sigma_{c} \\
x_{a} \sigma_{b} \sigma_{c} \sigma_{a}=\sigma_{a} \sigma_{b} \sigma_{c} x_{a} \\
\sigma_{a} \sigma_{b} x_{c} \sigma_{a}=\sigma_{b} x_{c} \sigma_{a} \sigma_{b} \\
x_{a} \sigma_{b} x_{c} \sigma_{a}=\sigma_{b} x_{c} \sigma_{a} x_{b}
\end{gathered}
$$


- Pseudocycle: if the edges $a_{1}, \ldots, a_{n}$ form an irreducible pseudocycle and if $a_{1}$ is not the start edge nor is $a_{n}$ the end edge of a reverse, then

$$
\sigma_{a_{1}} \cdots \sigma_{a_{n-1}}=\sigma_{a_{2}} \cdots \sigma_{a_{n}}, \quad x_{a_{1}} \sigma_{a_{2}} \cdots \sigma_{a_{n-1}}=\sigma_{a_{2}} \cdots \sigma_{a_{n-1}} x_{a_{n}} .
$$

A proof of Theorem 5.1 is given in [34]. The steps of the proof are the same as for Theorem 2.1.

The last aim of this section is to give graph presentations for the singular braid monoid on $n$ strands of the annulus.

THEOREM 5.2. The singular braid monoid on $n$ strands of the annulus $S B_{n}($ Ann) admits the following presentation:

- Generators: $\sigma_{i}, \sigma_{i}^{-1}, x_{i}, i=1, \ldots, n-1, \tau, \tau^{-1}$;

- Relations:

$$
\begin{array}{ll}
\text { (R1) } & \sigma_{i} \sigma_{j}=\sigma_{j} \sigma_{i} \quad \text { if }|i-j|>1 \\
\text { (R2) } & x_{i} x_{j}=x_{j} x_{i} \quad \text { if }|i-j|>1 \\
\text { (R3) } & x_{i} \sigma_{j}=\sigma_{j} x_{i} \quad \text { if }|i-j| \neq 1 \\
\text { (R4) } & \sigma_{i} \sigma_{i+1} \sigma_{i}=\sigma_{i+1} \sigma_{i} \sigma_{i+1} \\
\text { (R5) } & \sigma_{i} \sigma_{i+1} x_{i}=x_{i+1} \sigma_{i} \sigma_{i+1} \\
\text { (R6) } & \sigma_{i+1} \sigma_{i} x_{i+1}=x_{i} \sigma_{i+1} \sigma_{i} ; \\
\text { (R7) } & \tau \sigma_{1} \tau \sigma_{1}=\sigma_{1} \tau \sigma_{1} \tau \\
\text { (R8) } & \tau \sigma_{1} \tau x_{1}=x_{1} \tau \sigma_{1} \tau \\
\text { (R9) } & \tau \sigma_{i}=\sigma_{i} \tau \quad \text { if } i \geq 2 \\
\text { (R10) } & \tau x_{i}=x_{i} \tau \quad \text { if } i \geq 2 \\
\text { (R11) } & \sigma_{i} \sigma_{i}^{-1}=\sigma_{i}^{-1} \sigma_{i}=\tau \tau^{-1}=\tau^{-1} \tau=1 .
\end{array}
$$

Proof. The geometric interpretation of $\sigma_{i}$ and $\tau$ is given in Figure 13, and $\sigma_{i}^{-1}$ and $\tau^{-1}$ are their inverses. The generator $x_{i}$ corresponds to a singular crossing between strands $i$ and $i+1$.
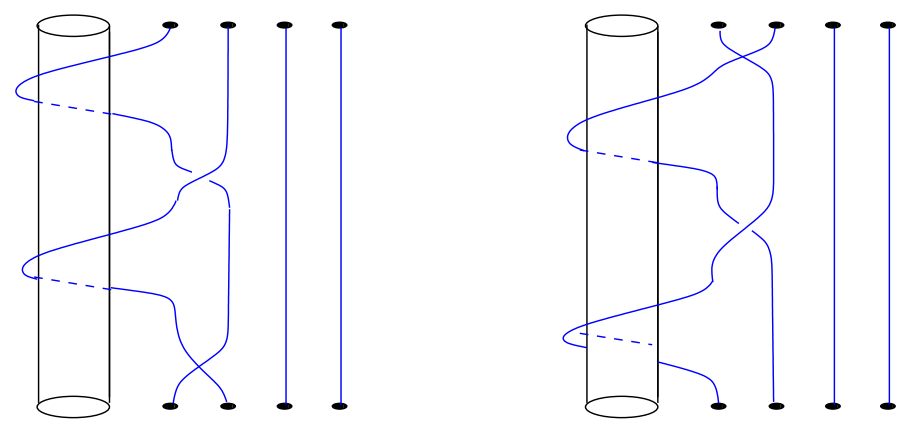

Fig. 17. The words $\tau \sigma_{1} \tau x_{1}$ and $x_{1} \tau \sigma_{1} \tau$ represent the same element in $S B_{n}(\mathrm{Ann})$ 
We obtain the Reidemeister moves for singular knot theory in a solid torus if we add the move depicted in Figure 17 to the regular (without singularities) Reidemeister moves of knot theory in a solid torus.

This Reidemeister move means how a singular point goes around the axis of the torus (fixed string). The proof that relations (R1)-(R11) give a complete list is standard: every isotopy can be decomposed into a sequence of elementary isotopies which correspond to relations (R1)-(R11) (see also [21]).

REMARK 5.3. The singular braid monoid on $n$ strands of the annulus differs from the singular Artin monoid of type $\mathcal{B}$ as defined by R. Corran [13], where the numbers of singular and regular generators are the same. The singular generator associated to $\tau$ cannot be interpreted geometrically as above.

As in Section 4 we consider 1-punctured graphs. To any edge $a$ disjoint from the distinguished vertex $v$ of $\Gamma$ we associate three singular braids: $\sigma_{a}$ will denote the positive half-twist associated to $a$ (as in Figure 1), $\sigma_{a}^{-1}$ the corresponding negative half-twist, and $\tau_{a}$ the corresponding singular crossing.

The graph presentations for the singular braid monoid in the solid torus arise from Theorems 5.1 and 5.2 (see [34]).

THEOREM 5.4. Let $\Gamma$ be a 1-punctured graph on $n$ vertices. The monoid $S B_{n}(\mathrm{Ann})$ admits the presentation $\left\langle X_{\Gamma}, R_{\Gamma}\right\rangle$, where

- $X_{\Gamma}=\left\{\sigma_{a}, \sigma_{a}^{-1}, x_{a}, \tau_{b}, \tau_{b}^{-1}\right\}$, for any edge a of $\Gamma$ not incident with the distinguished vertex $v$, and for any edge $b$ of $\Gamma$ adjacent to $v$;

- $R_{\Gamma}$ is formed by the relations given in Theorems 4.1 and 5.1 and the following new nodal and invertibility relations:

$$
\begin{gathered}
\sigma_{a} \tau_{b} \sigma_{c} x_{a}=x_{c} \sigma_{a} \tau_{b} \sigma_{c}, \\
\tau_{b} \sigma_{c} \sigma_{a} \tau_{b} x_{c}=x_{a} \tau_{b} \sigma_{c} \sigma_{a} \tau_{b}, \\
\tau_{b} \tau_{b}^{-1}=\tau_{b}^{-1} \tau_{b}=1 .
\end{gathered}
$$

\section{References}

[1] J. Baez, Link invariants of finite type and perturbation theory, Lett. Math. Phys. 26 (1992), 43-51.

[2] P. Bellingeri, Surface braid groups and polynomial link invariants, Thesis, Univ. Grenoble I, 2003.

[3] - On presentation of surface braid groups, J. Algebra 274 (2004), 543-563.

[4] P. Bellingeri and L. Funar, Braids on surfaces and finite type invariants, C. R. Acad. Sci. Paris 338 (2004), 157-162.

[5] D. Bessis, Groupes de tresses et éléments réguliers, J. Reine Angew. Math. 518 (2000), 1-40. 
[6] D. Bessis, F. Digne and J. Michel, Springer theory in braid groups and the BirmanKo-Lee monoid, Pacific J. Math. 205 (2002), 287-309.

[7] J. S. Birman, Braids, Links, and Mapping Class Groups, Ann. of Math. Stud. 82, Princeton Univ. Press, 1974.

[8] J. S. Birman, New points of view in knot theory, Bull. Amer. Math. Soc. 28 (1993), 253-287.

[9] J. S. Birman, K. H. Ko and S. J. Lee, A new approach to the word problem and conjugacy problems in the braid groups, Adv. Math. 139 (1998), 322-353.

[10] E. Brieskorn, Sur les groupes de tresses [d'après V. I. Arnol'd], Séminaire Bourbaki, 24ème année (1971/1972), Exp. No. 401, Lecture Notes in Math. 317, Springer, Berlin, 1973, 21-44.

[11] E. Brieskorn und K. Saito, Artin-Gruppen und Coxeter-Gruppen, Invent. Math. 17 (1972), 245-271.

[12] N. Bourbaki, Groupes et algèbres de Lie, Chaps. 4-6, Masson, Paris, 1981.

[13] R. Corran, A normal form for a class of monoids including the singular braid monoids, J. Algebra 223 (2000), 256-282.

[14] H. S. M. Coxeter and W. O. J. Moser, Generators and Relations for Discrete Groups, 2nd ed., Spinger, 1965.

[15] P. Deligne, Les immeubles des groupes de tresses généralisés, Invent. Math. 17 (1972), 273-302.

[16] M. Eisermann, Les invariants rationnels de type fini ne distinguent pas les noeuds dans $S^{2} \times S^{1}$, C. R. Acad. Sci. Paris 332 (2001), 51-55.

[17] E. Fadell and L. Neuwirth, Configuration spaces, Math. Scand. 10 (1962), 111-118.

[18] E. Fadell and J. Van Buskirk, The braid groups of $E^{2}$ and $S^{2}$, Duke Math. J. 29 (1962), 243-258.

[19] J. González-Meneses, New presentations of surface braid groups, J. Knot Theory Ramif. 10 (2001), 431-451.

[20] - , Ordering pure braid groups on closed surfaces, Pacific J. Math. 203 (2002), 369-378.

[21] Presentations for the monoids of singular braids on closed surfaces, Comm. Algebra 30 (2002), 2829-2836.

[22] J. González-Meneses and L. Paris, Vassiliev invariants of surface braid groups, Trans. Amer. Math. Soc. 356 (2004), 219-243.

[23] J. Guaschi and P. Gonçalves, On the structure of surface pure braid groups, J. Pure Appl. Algebra 182 (2003), 33-64.

[24] J. W. Han and K. H. Ko, Positive presentations of the braid groups and the embedding problem, Math. Z. 240 (2002), 211-232.

[25] E. Irmak, N. V. Ivanov, and J. D. McCarthy, Automorphisms of surface braid groups, math.GT/0306069.

[26] S. Lambropoulou, Knot theory related to generalized and cyclotomic Hecke algebras of type $\mathcal{B}$, J. Knot Theory Ramif. 8 (1999), 621-658.

[27] L. Paris, Birman's conjecture on singular braids on closed surfaces, ibid. 13 (2004), 895-915.

[28] L. Paris and D. Rolfsen, Geometric subgroups of surface braid groups, Ann. Inst. Fourier (Grenoble) 49 (1999), 417-472.

[29] V. Sergiescu, A direct approach to the planar graph presentations of the braid group, in: Singularities of Holomorphic Vector Fields and Related Topics, Proc. RIMS Kyoto 878 (1994), 103-107.

[30] - Graphes planaires et présentation des groupes de tresses, Math. Z. 214 (1993), 477-490. 
[31] R. Skora, Closed braids in 3-manifolds, ibid. 211 (1992), 173-187.

[32] V. V. Vershinin, Braid groups and loop spaces, Russian Math. Surveys 54 (1999), 273-350; transl. from Uspekhi Mat. Nauk 54 (1999), no. 2, 3-84.

[33] —, On the singular braid monoid, ArXiv:mathGR/0309339.

[34] —, Présentations de Sergiescu pour les généralisations de tresses, preprint.

[35] O. Zariski, On the Poincaré group of rational plane curves, Amer. J. Math. 58 (1936), 607-619.

[36] - , The topological discriminant group of a Riemann surface of genus $p$, ibid. 59 (1937), 335-358.

Dipartimento di Matematica

Università di Pisa

Largo B. Pontecorvo 5

56127 Pisa, Italy

E-mail: bellingeri@mail.dm.unipi.it
Département des Sciences Mathématiques Université Montpellier II Place Eugène Bataillon 34095 Montpellier Cedex 5, France E-mail: vershini@math.univ-montp2.fr

Sobolev Institute of Mathematics Novosibirsk, 630090, Russia E-mail: versh@math.nsc.ru

Received 25 June 2004;

in revised form 12 June 2005 